\title{
Angle-resolved transmission spectroscopy of three-dimensional photonic crystals fabricated by direct laser writing
}

\author{
M. Deubel ${ }^{\mathrm{a})}$ and M. Wegener \\ Institut für Angewandte Physik, Wolfgang-Gaede-Straße 1, Universität Karlsruhe (TH), \\ D-76131 Karlsruhe, Germany \\ S. Linden and G. von Freymann \\ Institut für Nanotechnologie, Forschungszentrum Karlsruhe in der Helmholtz-Gemeinschaft, Postfach 3640, \\ D-76021 Karlsruhe, Germany
}

(Received 17 May 2005; accepted 5 October 2005; published online 22 November 2005)

\begin{abstract}
Oblique incidence optical transmission spectra of three-dimensional photonic crystal templates, i.e., woodpile structures, are presented. They are compared with transmission spectra calculated using a scattering-matrix approach. In addition, band-structure calculations are presented. The comparison reveals intricate details of the three-dimensional photonic band structure demonstrating the high overall sample quality. (C) 2005 American Institute of Physics. [DOI: 10.1063/1.2137899]
\end{abstract}

Direct laser writing (DLW) (Refs. 1 and 2) has recently been proven to be a powerful tool for the fabrication of highquality three-dimensional photonic crystal templates at telecommunication wavelengths. ${ }^{3-5}$ More recently, we have shown ${ }^{6}$ that such photoresist templates indeed can be translated into high-index silicon structures via a double-inversion procedure using chemical-vapor deposition (CVD). These silicon structures essentially have the same quality as the templates. Thus, DLW combined with subsequent CVD filling appears to be a very attractive alternative to approaches using electron-beam lithography and sophisticated stacking procedures $^{7,8}$ or to silicon inverse opals. ${ }^{9,10}$

This brings us to the critical question: How good are the three-dimensional photoresist templates made by DLW really? Presently, the most powerful method for the assessment of the actual optical quality is optical spectroscopy. Our corresponding work on woodpile (layer-by-layer) structures has revealed a very high quality indeed, however, scattering losses still appeared to play a certain role. ${ }^{3}$ This was essentially concluded from the fact that, for "normal incidence," the measured transmission energetically above the fundamental stop band did not come back to $100 \%$ as naively expected. We will see below that this overall conclusion was incorrect. It is the aim of this paper to present improved data on the optical transmission of these photonic crystals for normal incidence as well as for oblique incidence, using a dedicated setup which allows to perform spectroscopy on finite samples and, at the same time, minimizes the opening angle of light impinging onto the sample. The comparison of the observed intricate fine structure with theory, evaluated for the identical (finite) structures, allows us to conclude that these photonic crystals come very close to the ideal. This, in our opinion, raises new hopes that inexpensive large-scale high-quality three-dimensional photonic band-gap materials at telecommunication wavelengths - with or without additionally incorporated defects and/or waveguide structureswill become readily available in the near future.

In DLW, femtosecond laser pulses are tightly focused into a photoresist. Only in the immediate focal volume is the photoresist exposed via multiphoton absorption. After the de-

\footnotetext{
${ }^{a)}$ Electronic mail: markus.deubel@physik.uni-karlsruhe.de $-\theta$ agree within the reproducibility of the measurements, inEVA-STAR (Elektronisches Volltextarchiv - Scientific Articles Repository)

velopment, only the exposed regions remain. Scanning the resist in three dimensions with respect to the fixed laser focus allows writing of essentially arbitrary three-dimensional structures. With a numerical aperture (NA) of the focusing lens $\mathrm{NA}=1.4$, the commercial photoresist SU-8, and pulses from an amplified laser system (Spectra Physics Hurricane), we routinely achieve a lateral resolution of $150 \mathrm{~nm}$. Here, the famous woodpile structure ${ }^{11}$ is fabricated, which is especially well suited for DLW. To achieve the desired diamondderived structure, the lattice constant $c$ is chosen as $c=\sqrt{2} a$, with the rod spacing $a$. Structures with rod spacings from 0.65 to $1.0 \mu \mathrm{m}$ have been fabricated; here we only show data for one $a=1.0 \mu \mathrm{m}$ sample. Corresponding electron micrographs and further details on the fabrication have been published in Ref. 3. The sample sits on a glass substrate, has a total area of $(100 \mu \mathrm{m})^{2}$, and contains 24 layers. Its filling fraction is approximately $27 \%$.

In our dedicated home-built oblique-incidence optical setup, we use a standard 100-W halogen lamp, which is coupled into an optical multimode fiber $(200-\mu \mathrm{m}$ core diameter), the output of which is sent through a Glan-Thompson polarizer and is imaged onto the sample with an estimated effective half-opening angle of five degrees. The light transmitted by the sample is spatially filtered in an intermediate image plane (which can also be inspected with a camera system) in order to exclusively collect light that has actually passed the sample. This aspect is especially important for large angles of incidence, $\theta$, with respect to the surface normal. The filtered light is imaged onto a second multimode optical fiber connected to a home-built Fourier-transform spectrometer, which gives access to the spectral range from 600 to $2200 \mathrm{~nm}$ wavelength. To ensure proper normalization, the measured transmission spectra are normalized to the transmission of the bare glass substrate for the same angle of incidence (requiring only a very small lateral movement of the sample). This implies that the transmission defined in this manner can slightly exceed unity for wavelengths and/or angles where the photonic crystal essentially acts as an antireflection coating of the glass substrate. Spectra are taken from $0^{\circ}$ (normal incidence) to $60^{\circ}$ in steps of $5^{\circ}$. We only show data for one direction, because the spectra for $+\theta$ and 


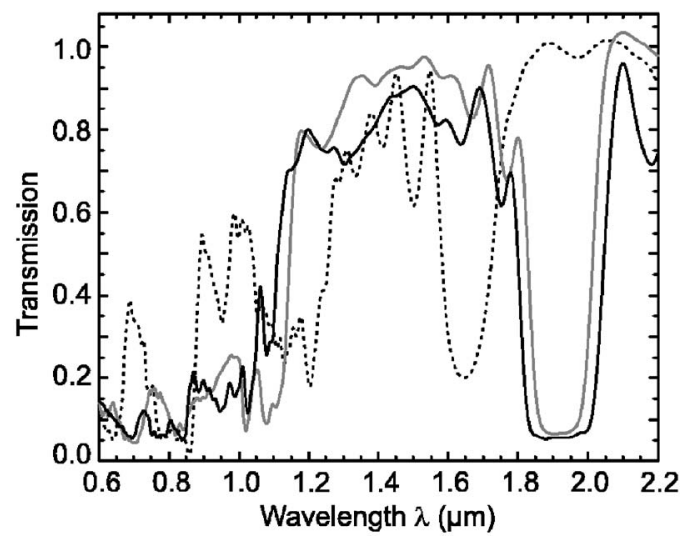

FIG. 1. Measured transmission spectra of a woodpile structure with $a$ $=1.0 \mu \mathrm{m}$ for normal incidence, $p$ polarization (dotted line), and for oblique incidence $\left(\theta=60^{\circ}\right)$; in $s$ (solid black line) and $p$ polarization (solid gray line), respectively. All spectra are normalized to transmission of the bare substrate (see discussion in the main text).

dicating that possible misalignments of the rods are negligible. We take spectra for an incident linear polarization in the plane of incidence ( $p$ polarization) and perpendicular to it ( $s$ polarization).

Figure 1 shows three examples of measured transmission spectra. All spectral features are reproducible; the noise is less than the linewidth of the plot. Notably, the fundamental stop band for an angle of, e.g., $60^{\circ}$ is very pronounced, with steep edges and low transmission in the stop band. The transmission on the long- and on the short-wavelength side of this stop band reaches values close to $100 \%$. Also, pronounced Fabry-Perot fringes are observed energetically above the stop band, another first indicator of high sample quality. In order to get an overview, we convert the set of measured spectra into a gray-scale plot, where the transmission is depicted as a function of angle of incidence and wavelength. The orientation of the plot is chosen to allow for better comparison with band-structure calculations (below). On the gray scale, dark areas correspond to low transmission while bright areas to high transmission-allowing for an intuitive interpretation. The corresponding images in Fig. 2 reveal a rich fine structure. For normal incidence, the spectra are not quite identical because the rod direction for the uppermost layer is parallel (orthogonal) to the electric field in $s$ polarization ( $p$ polarization). In other words, we observe an influence of the surface termination on the optical properties. By increasing the angle of oblique incidence, the fundamental stop band shifts to the blue, hits another shifting structure, and eventually shifts to the red end of the spectrum. Note that the details of this crossing scenario are different for the two linear polarizations.

To allow for a direct comparison with the expectations for a perfect structure, and to interpret the observed structures, we perform both scattering-matrix calculations ${ }^{12,13}$ and band-structure calculations (MIT Photonic-Bands Package $\left.^{14}\right)$. We use the actual woodpile structure described above; i.e., we incorporate the ellipticity of the rods, the overlap of orthogonal rods, use the refractive index of SU-8 ( $n=1.57), a=1.0 \mu \mathrm{m}, c=\sqrt{2} a$, a volume filling fraction of $27 \%, 24$ layers on a semi-infinite glass substrate $(n=1.52)$, and the same pragmatic definition of "transmission" as in the experiment (see above) for the scattering-matrix calculations. This also means that we only "collect" the zeroth transmisThis also means that we only "collect" the zeroth transmis- sion spectra.
Downloaded 18 Mar 2009 to 129.13.72.198. Redistribution subject to AlP license or
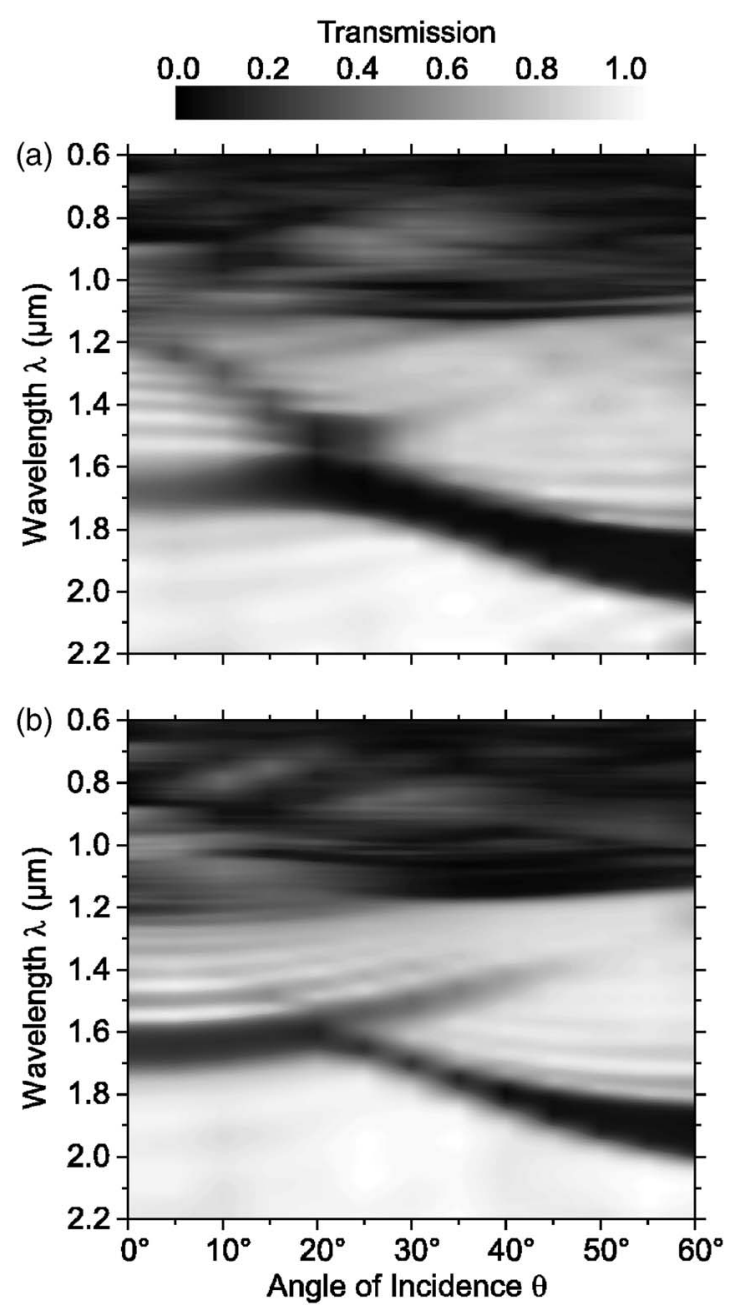

FIG. 2. Gray-scale plot of the measured transmission (compare Fig. 1) vs angle of incidence and wavelength. (a) $s$ polarization, (b) $p$ polarization.

sion order; the higher diffracted orders (which are also calculated) must not be incorporated. In our above experiment, they are not collected by the optics. Still, the diffracted orders take away energy from the zeroth transmission order, hence, reducing the transmission coefficient. This can be a very efficient "channel" and must not be confused with scattering losses. The results of the scattering-matrix calculations are summarized in Fig. 3, and those of the band-structure calculation in Fig. 4, where only the relevant part of the Brillouin zone is shown. Notably, the gray-scale plots of the calculated transmission (Fig. 3) and those of the measured transmission (Fig. 2) agree very well. This is also true for the polarization dependence. Small quantitative deviations are due to the finite opening angle of our optics and imperfections still present in the sample. The comparison with the photonic band structure (Fig. 4) gives additional insight. Starting from the $\Gamma \mathrm{X}^{\prime}$ direction for normal incidence, the fundamental stop band shifts to the blue when going towards the $\Gamma U^{\prime}$ direction for oblique incidence. At the same time, higher bands (see gray area) shift to the red and meet the fundamental stop band at the $\mathrm{U}^{\prime}$ point. By further increasing the angle, the shift directions reverse. These features are also seen in the measured (Fig. 2) and calculated (Fig. 3) transmission spectra. Below about $1.1 \mu \mathrm{m}$ wavelength, many additional bands occur, leading to highly structured transmis-

to AIP license or copyright; see http://apl.aip.org/apl/copyright.jsp 

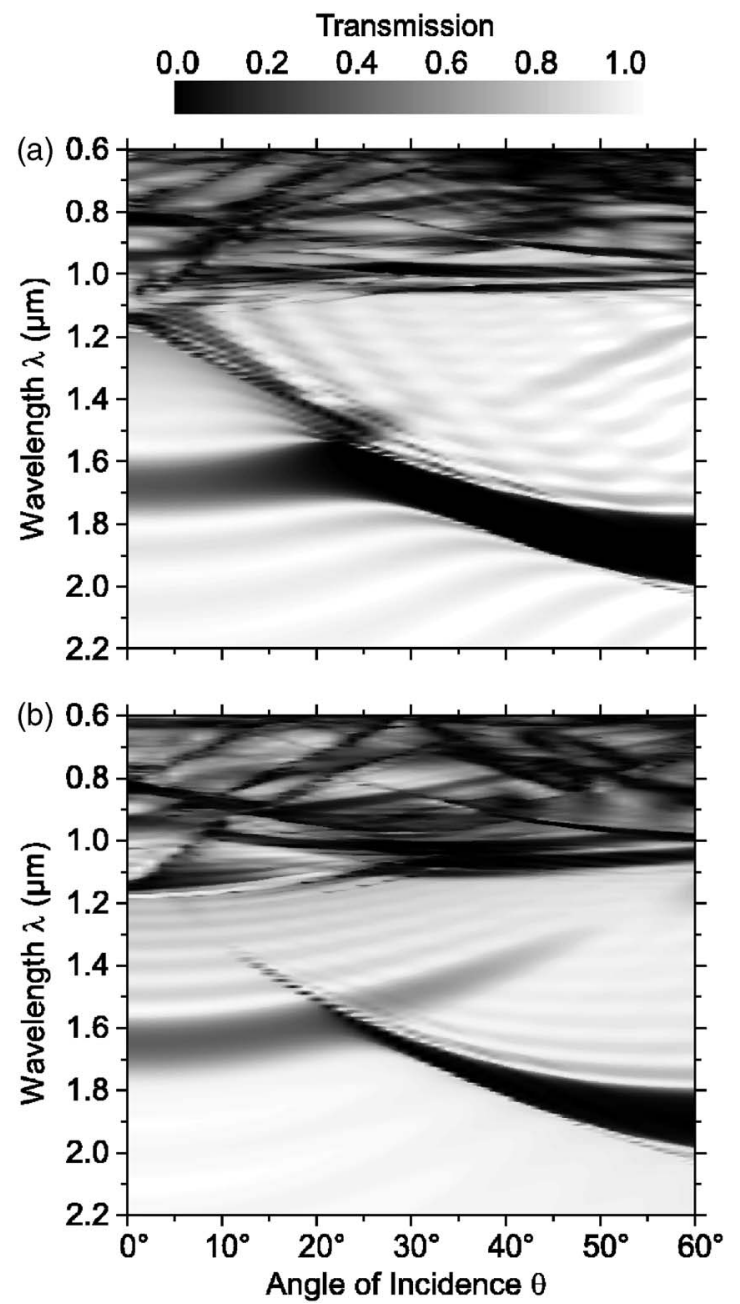

FIG. 3. Gray-scale plot of the calculated transmission vs angle of incidence and wavelength. The woodpile structure corresponds to that of the experiment (Fig. 2). (a) $s$ polarization, (b) $p$ polarization.

In conclusion, we have measured transmission spectra of three-dimensional woodpile photonic crystal templates with stop bands in the telecommunication regime under oblique incidence. The comparison with scattering-matrix calculations and with band-structure calculations gives excellent agreement, indicating an excellent quality of our structures. From our data it becomes obvious that one must be very cautious in interpreting the detailed line shapes of spectra acquired with large opening angles of the incident light, which tends to smear out all spectral features, thus, mimicking lower sample quality.

The authors acknowledge support by the Deutsche Forschungsgemeinschaft (DFG) and by the state of Baden-

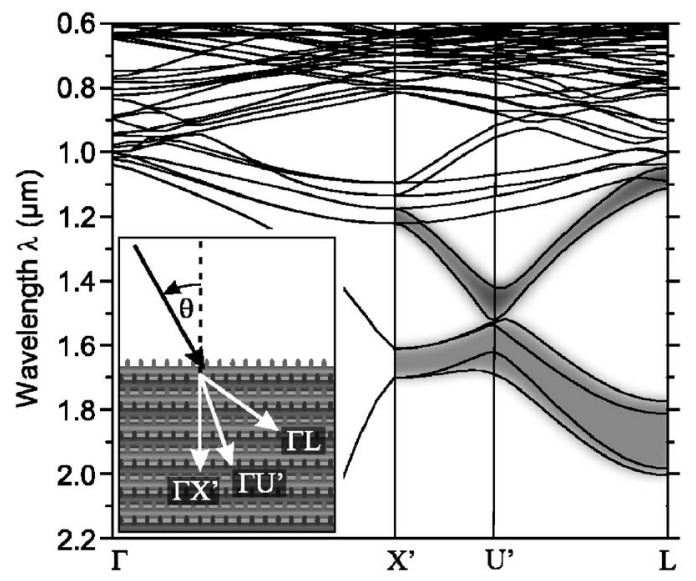

FIG. 4. Calculated band structure of the woodpile structure investigated in Figs. 1-3. The gray areas are guides to the eye, aiming at simplifying the comparison with Figs. 2 and 3. The inset illustrates characteristic propagation directions.

Württemberg through subproject A1.4 of the DFGForschungszentrum "Functional Nanostructures" (CFN) and by projects DFG-We 1497/9-1 and DFG-Fr 1671/4-3 (Emmy Noether-program).

${ }^{1}$ S. Maruo, O. Nakamura, and S. Kawata, Opt. Lett. 22, 132 (1997).

${ }^{2}$ B. H. Cumpston, S. P. Ananthavel, S. Barlow, D. L. Dyer, J. E. Ehrlich, L. L. Erskine, A. A. Heikal, S. M. Kuebler, I.-Y. S. Lee, D. McCordMaughon, J. Qin, H. Röckel, M. Rumi, X.-L. Wu, S. R. Marder, and J. W. Perry, Nature (London) 398, 51 (1999).

${ }^{3}$ M. Deubel, G. von Freymann, M. Wegener, S. Pereira, K. Busch, and C. M. Soukoulis, Nat. Mater. 3, 444 (2004).

${ }^{4}$ M. Deubel, M. Wegener, A. Kaso, and S. John, Appl. Phys. Lett. 85, 1895 (2004).

${ }^{5}$ K. K. Seet, V. Mizeikis, S. Matsuo, S. Juodkazis, and H. Misawa, Adv. Mater. (Weinheim, Ger.) 17, 541 (2005).

${ }^{6}$ N. Tétreault, G. von Freymann, M. Deubel, M. Hermatschweiler, F. PérezWillard, S. John, M. Wegener, and G. A. Ozin, Adv. Mater. (Weinheim, Ger.) (in press).

${ }^{7}$ S. Y. Lin, J. G. Fleming, D. L. Hetherington, B. K. Smith, R. Biswas, K. M. Ho, M. M. Sigalas, W. Zubrzycki, S. R. Kurtz, and J. Bur, Nature (London) 394, 251 (1998).

${ }^{8}$ S. Noda, K. Tomoda, N. Yamamoto, and A. Chutinan, Science 289, 604 (2000).

${ }^{9}$ A. Blanco, E. Chomski, S. Grabtchak, M. Ibisate, S. John, S. W. Leonard, C. Lopez, F. Meseguer, H. Miguez, J. P. Mondia, G. A. Ozin, O. Toader, and H. M. van Driel, Nature (London) 405, 437 (2000).

${ }^{10}$ Y. A. Vlasov, X.-Z. Bo, J. C. Sturm, and D. J. Norris, Nature (London) 414, 289 (2001).

${ }^{11}$ K. M. Ho, C. T. Chan, C. M. Soukoulis, R. Biswas, and M. Sigalas, Solid State Commun. 89, 413 (1994).

${ }^{12}$ D. M. Whittaker and I. S. Culshaw, Phys. Rev. B 60, 2610 (1999).

${ }^{13}$ S. G. Tikhodeev, A. L. Yablonskii, E. A. Muljarov, N. A. Gippius, and T. Ishihara, Phys. Rev. B 66, 045102 (2002).

${ }^{14}$ S. G. Johnson and J. D. Joannopoulos, Opt. Express 8, 173 (2001). 